\title{
Experimental Evaluation of the VEM Drive
}




\begin{tabular}{||c|c|c|l||}
\hline \multicolumn{5}{||c||}{ DOCUMENT CHANGE RECORD } \\
\hline Project: Experimental Results Reproduction Regarding the VEM Drive \\
\hline \multicolumn{1}{||c||}{ Change Status Log: } \\
\hline Issue: & CN No.: & Class & Reason for Change / Pages Affected \\
\hline 1 & $\cdots$ & $\cdots$ & Initial Issue \\
\hline & & & \\
\hline Changes between the current and the previous issue are marked with a bar on the left side or deleted \\
\hline $\begin{array}{l}\text { Page Status Log: } \\
\text { This Document comprises the following pages at issues shown: }\end{array}$ \\
\hline Pages/lssue \\
\end{tabular}




\section{Table of Contents}

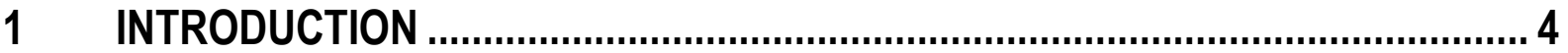

2 EQUIPMENT

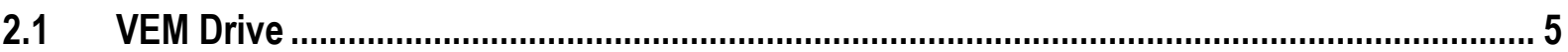

2.1.1 Mechanical Components 5

2.1.2 Electrical Components 6

2.1.3 Electrical Setup \& Calibration 7

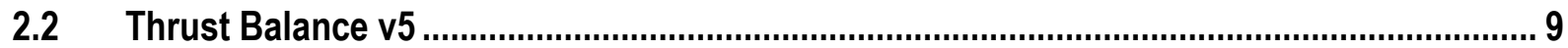

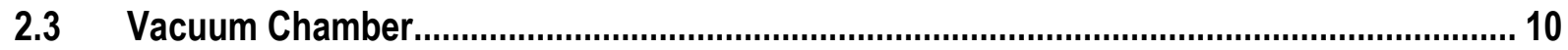

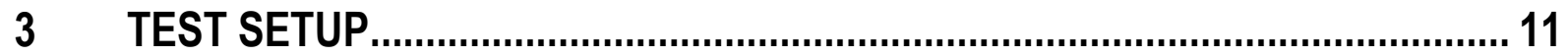

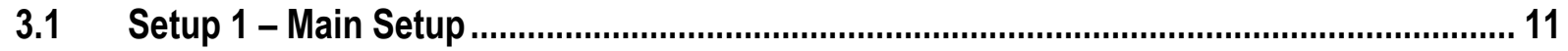

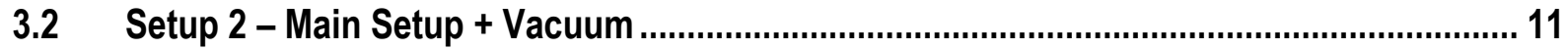

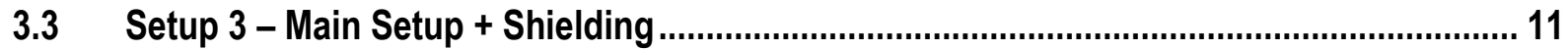

3.3.1 Setup 3a - Metal Shielding 11

3.3.2 Setup 3b - Thermal Shielding 12

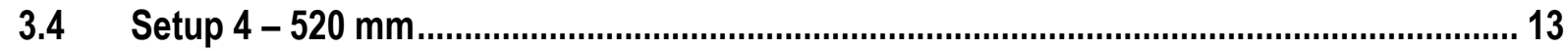

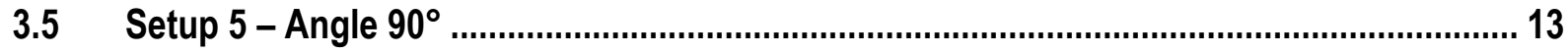

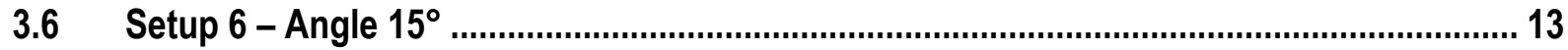

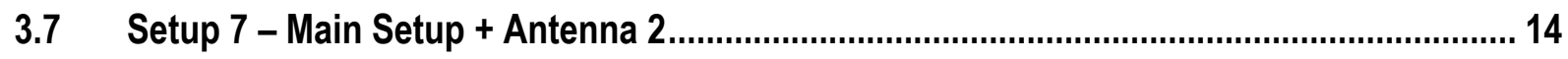

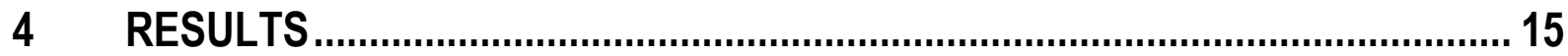

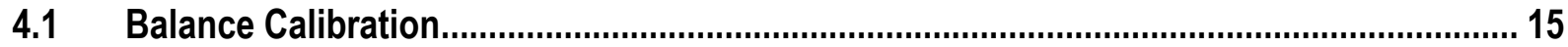

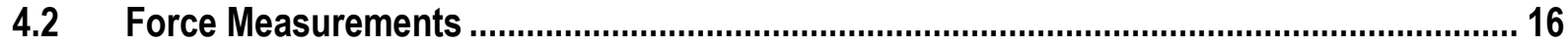

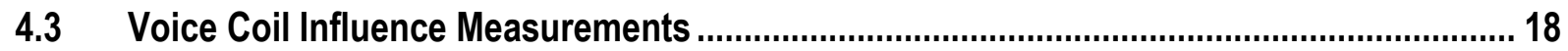

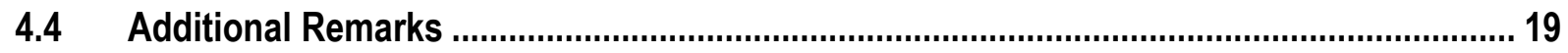

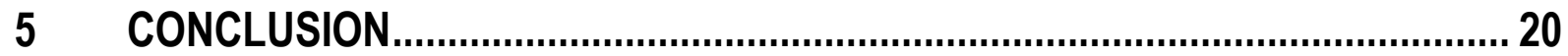

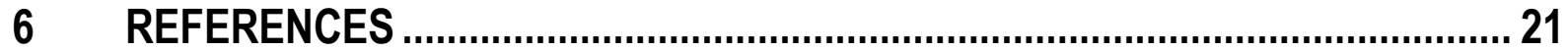




\section{INTRODUCTION}

The VEM Drive (Variable-Electro-Magnetic Drive) is an invention by Space Warp Dynamics LLC that claims to distort space-time and thus creating an attractive force upon a target using frequencies in the VHF band.

In their paper [1] they tested an antenna facing a torsion balance with different target materials. The balance used a wire of unknown material as a torsional spring and was housed inside a faraday cage and a plastic container. The deflection of the balance was measured using a laser that reflected from a mirror mounted on the balance. The antenna and the cage were in air. They mainly present swing rates of the balance (which is a useless measurement given the unknown wire of the balance). At the end, they present a couple of force values shown in Table 1. The target material and the distance between the drive and target is not specified for these values.

\begin{tabular}{|l|l|}
\hline Power [W] & Force [N] \\
\hline 175 & 0.63 \\
\hline 375 & 0.71 \\
\hline 650 & 1.25 \\
\hline
\end{tabular}

Table 1: Force values for different power levels

This test campaign tries to replicate these results with a more accurate torsion balance at lower power levels. Two different VEM antenna were used. The antenna was put inside a large vacuum chamber to sufficiently shield any electromagnetic radiation to comply with regulations. $A \mu \mathrm{N}$ resolution (mostly metallic) thrust balance was used in order to measure thrust. A large non-metal POM plate, placed on the balance, was used as a target. 


\section{EQUIPMENT}

In this section the equipment for the test campaign is described.

\subsection{VEM Drive}

This section describes the VEM Drive. It is split into two subsection. The first describes the antenna that is the VEM drive and its mounting stand. The second subsection describes all electrical components and the radio frequency (RF) setting used during the test campaign.

\subsubsection{Mechanical Components}

The VEM Drive itself consists of three main assemblies. The first is a fractal antenna made out of four copper coated aluminium plates (Figure 1a) - a second antenna with a rotated fractal configuration was tested too. Two plates are connected to one N-Type connector (one to the centre pin, the other to the shield).

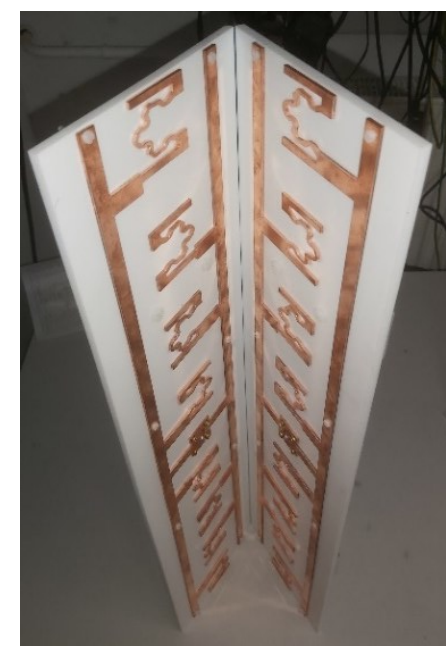

a) Antennas screwed to the PTFE plates

b) Back side of VEM Drive
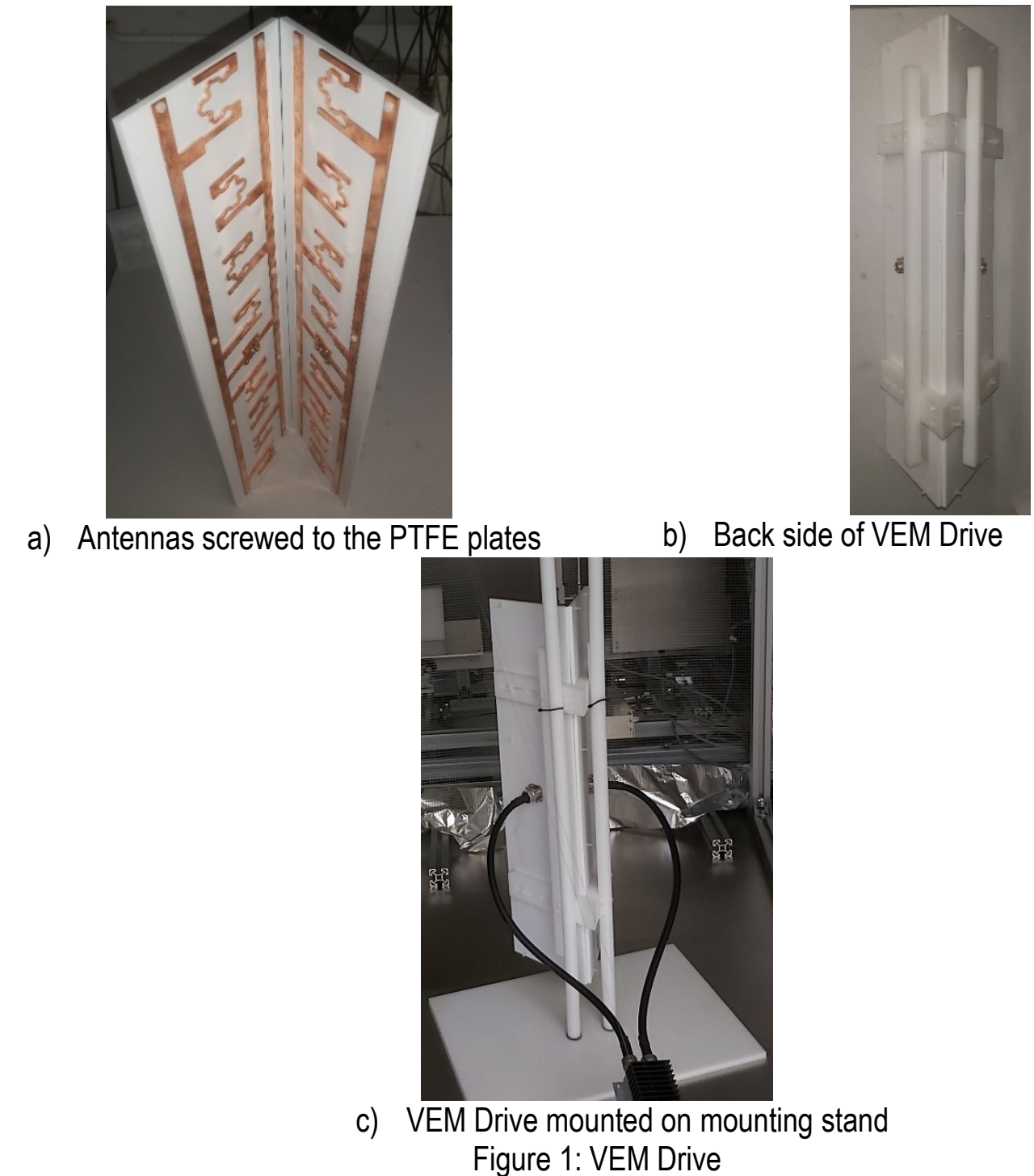
The second assembly consists of two polytetrafluoroethylene (PTFE) plates, each $5 \mathrm{~mm}$ thick. They are fixed to two Polyoxymethylene (POM) fixtures that combined assure an angle of $60^{\circ}$ between the PTFE plates. The last two parts of this assembly are two bars with a thickness of $16 \mathrm{~mm}$ and a length of $487 \mathrm{~mm}$ (Figure 1b).

The antenna assembly is mounted on the PTFE plates using nylon screws and nuts. The last assembly makes up the mounting stand for the VEM Drive. It consists of a $450 \mathrm{~mm} \times 295 \mathrm{~mm} \times 10 \mathrm{~mm}$ POM base plate with two $\varnothing 20 \mathrm{~mm} \times 1000 \mathrm{~mm}$ POM rods glued to it. The rods have notches in it. The VEM drive is mounted to the stand via zip ties that fit into the notches of the rods (Figure 1c).

\subsubsection{Electrical Components}

To deliver electromagnetic power in the desired frequency- and power-range, a setting consisting of RFcommercial-of-the-shelf products was used. A matching circuit was designed and provided by $\mathrm{Dr}$. Beukman. After analysing the antenna in our setup, a resonance frequency of $146 \mathrm{MHz}$ was selected for all measurements.

The following electronical and/or RF-components and devices were used:

\section{Rigol Frequency Generator DSG830}

Frequency range: $\quad 9 \mathrm{kHz}$ to $3 \mathrm{GHz} ; 0.01 \mathrm{~Hz}$ resolution

Power level: $\quad-11 \mathrm{dBm}$ to $13 \mathrm{dBm}$

Connector: $\quad \mathrm{N}-\mathrm{Type}-50 \Omega$

LAN controlled

\section{Minicircuits Amplifier ZHL-50W-52-S+}

Frequency range: $\quad 50 \mathrm{MHz}$ to $500 \mathrm{MHz}$

Power level:

Connector:

Output $47 \mathrm{dbm} ; 50 \mathrm{~W}$; gain $52 \mathrm{~dB}$

SMA-50 $\Omega$

\section{Minicircuits Power detector ZX47-40LN-S+}

Frequency range: $\quad 10 \mathrm{MHz}$ to $8,000 \mathrm{MHz}$

Frequency range: $\quad-50 \mathrm{dBm}$ to $+20 \mathrm{dBm}$

Connector: $\quad$ SMA-50 $\Omega$

\section{Minicircuits Bi-Directional Coupler ZFBDC20-62HP+}

Frequency range: $\quad 10 \mathrm{MHz}$ to $600 \mathrm{MHz}$

Power level: $\quad 47 \mathrm{dBm} ; 50 \mathrm{~W}$

Connector: $\quad$ SMA-50 $\Omega$

\section{UIY Circulator CC6060A}

Frequency range: $\quad 136 \mathrm{MHz}-174 \mathrm{MHz}$

Power level: $\quad 50 \mathrm{dBm} ; 100 \mathrm{~W}$

Connector: $\quad$ SMA-50 $\Omega$ 


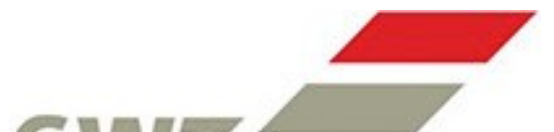

forschung+innovation
Doc: VEM-GWT-TN-1001

Issue: 1

Date: 5th March 2020

\section{Minicircuits Attenuators}

Frequency range: $\quad 0 \mathrm{MHz}-18,000 \mathrm{MHz}$

Connector:

SMA-50 $\Omega$; N-Type-50 $\Omega$

Attenuation:

BW-S40W20+ $40 \mathrm{~dB} @ 20 \mathrm{~W} ; 43 \mathrm{dBm}$

BW-N30W20+ $30 \mathrm{~dB} @ 20 \mathrm{~W} ; 43 \mathrm{dBm}$

BW-40N100W+40 dB @ 100 W; 50 dBm

BW-S20W2+ $20 \mathrm{~dB} @ 2 \mathrm{~W} ; 33 \mathrm{dBm}$

\section{Minicircuits $50 \Omega$ Coaxial Cables}

Frequency range: $\quad 0 \mathrm{MHz}-18,000 \mathrm{MHz}$

Power level:

Connectors:

min. 211 W @ $500 \mathrm{MHz}$

SMA \& N-Type

\section{Measurement devices}

Anritsu MS46122A Dual Port Vector Network Analyser

VNA for measuring reflected power to tune the matching device to minimum reflected power.

\section{Seeedstudio RF Explorer 3G}

Spectrum Analyser for validating generated frequency and to compare radiation level to other RF sources.

\subsubsection{Electrical Setup \& Calibration}

First the emitter was placed and aligned inside of the vacuum chamber $1130 \mathrm{~mm}$ next to the thrust balance with its POM target. The emitter antenna was connected to two coaxial N-Type-cables and so to a signalsplitter/combiner which was also provided with the emitter antenna. A SMA-N-Type-adapter connected the splitter/combiner to a coaxial cable (36") leading to the (floating) SMA-coaxial vacuum chamber feedthrough. Here the RF-behavior of the emitter and power leading cables was determined with the calibrated VNA.

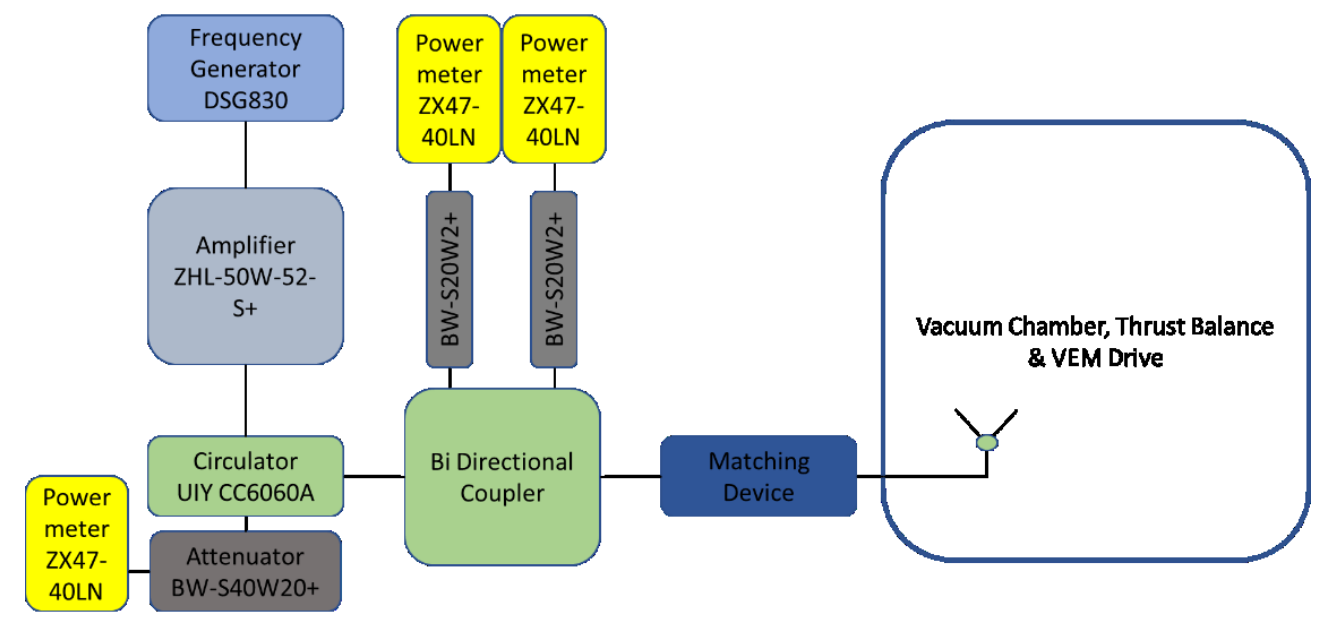

Figure 2: Electrical configuration 1 
Now the matching device was connected with a coaxial cable to the outside of the SMA-coaxial vacuumchamber feedthrough. Then, the VNA was connected to the input port of the matching device and the reflected power was minimized by tuning the devices capacitors with use of a Smith-chart-visualization. For first calibration measurements, the matching circuit was now connected as seen in configuration 1 (Figure 2).

Configuration 1 consisted of a frequency generator, a RF-amplifier, a circulator (with attached $40 \mathrm{~dB}$ attenuator and power detector to protect the amplifier from reflected power), a bi-directional-coupler (with attenuators and power detectors to measure input and reflected power), the matching device and connecting cables. The frequency generator was controlled via Ethernet connection with a laboratory computer and the power detectors were read out with a Labjack T7 device connected via USB to the same laboratory computer. The thrust balance was controlled by this computer as well. A Labview program allowed signal commanding, receiving and processing as well as statistical analysis of the input and output data.

Early measurement runs showed a difference between the commanded and the measured power which lead to a verification with a $20 \mathrm{~W} / 40 \mathrm{~dB}$ attenuator and a termination instead of matching device and emitter antenna. Also the behavior of the used power detectors was validated and the control algorithm was adapted. Nevertheless, inconsistent power detector signals were measured at power levels above $20 \mathrm{~W}$. To avoid this effects, the power detector for reflected power was replaced with a $50 \Omega$-termination which lead to even worse power measurements.

Configuration 2 (Figure 3) did not use the bi-directional coupler, because it was assumed, that the change in wave impedance due to thermal shifts led to unclear false-signals. This new configuration only measured the reflected power coming back from the emitter antenna.

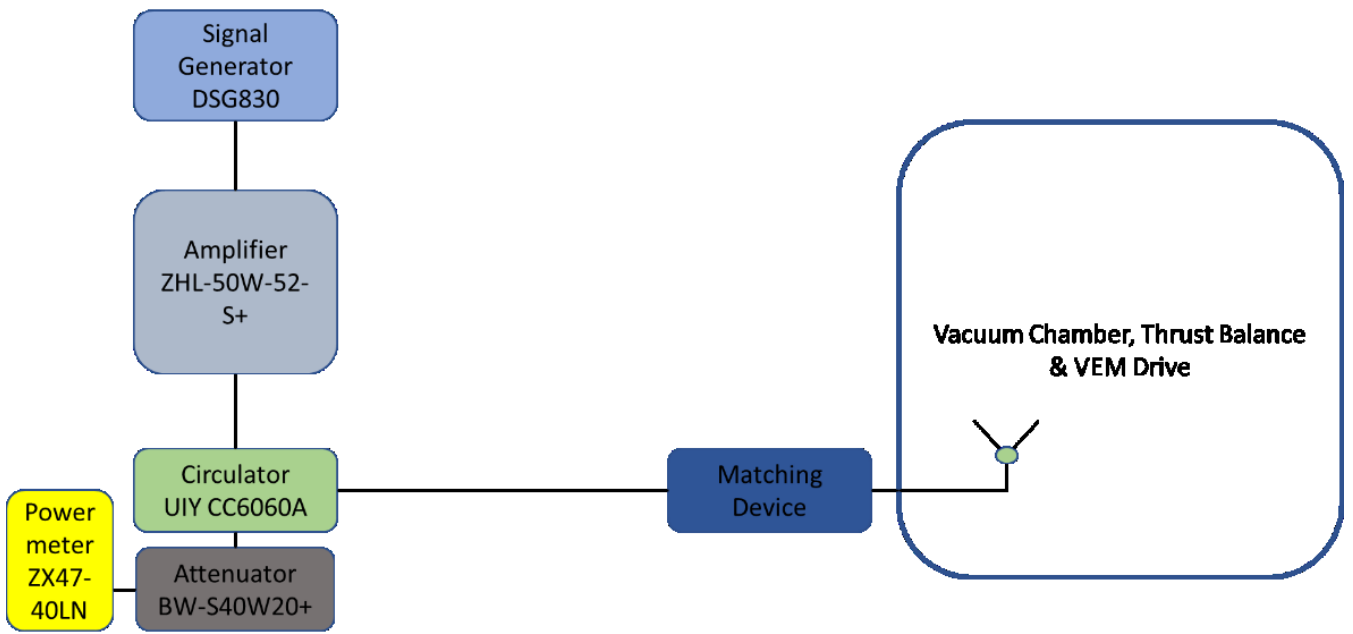

Figure 3: Electrical configuration 2

To verify the input power, the matching device and emitter were replaced with a $100 \mathrm{~W} / 40 \mathrm{~dB}$ attenuator and an attached power detector. This matched load showed the commanded and amplified power with only a small difference to the expected power. This configuration 2 was used throughout all tests.

During measurements at different power levels, the radiated RF-signal outside of the vacuum chamber was measured with a spectrum analyzer. 
Impedance matching was validated via VNA after measurement sequences and was adjusted if changes in the experimental setting were made (add FSX shield, add wire mesh).

\subsection{Thrust Balance v5}

The thrust balance (Figure 4), a so-called torsion balance, is described in reference [2] in detail. It uses two E-20 pivots by C-Flex as a torsional spring in the center, has an eddy current brake used to damp the oscillation and a voice coil to calibrate the balance. Furthermore, the bottom pivot is mounted on a rotational motor that can apply a torque onto this pivot that changes the resting position of the balance. This is used to align the voice coil, even if the balance is inside a vacuum chamber. For this test campaign, the shielding plates of the experiment box were removed and a target, made out of POM, weighing $620 \mathrm{~g}$, was placed on it instead. The electronics box underwent no changes.

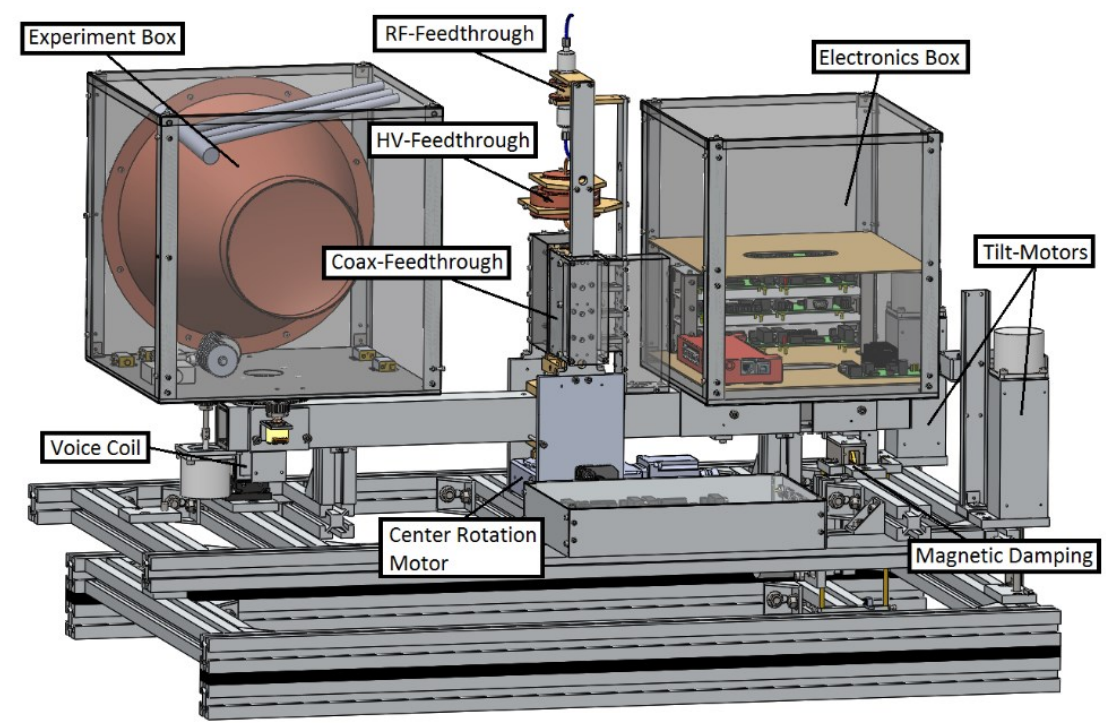

Figure 4: Torsion balance v5 [2]

The voice coil is used to produce a defined force, which is independent of the actual balance position (within a certain range of a few millimeters). This is used to actually calibrate the balance: A defined force causes a deflection of the balance, which is then further used to determine arbitrary forces. Such a voice coil is shown in Figure 5. It consists of a housing with a permanent magnet in the middle (mounted on the balance arm) and a moveable coil. This coil is connected to a precision power supply and produces a defined force proportional to the applied current. As we will see later, this coil can pick of electromagnetic radiation and cause anomalous force measurements. 

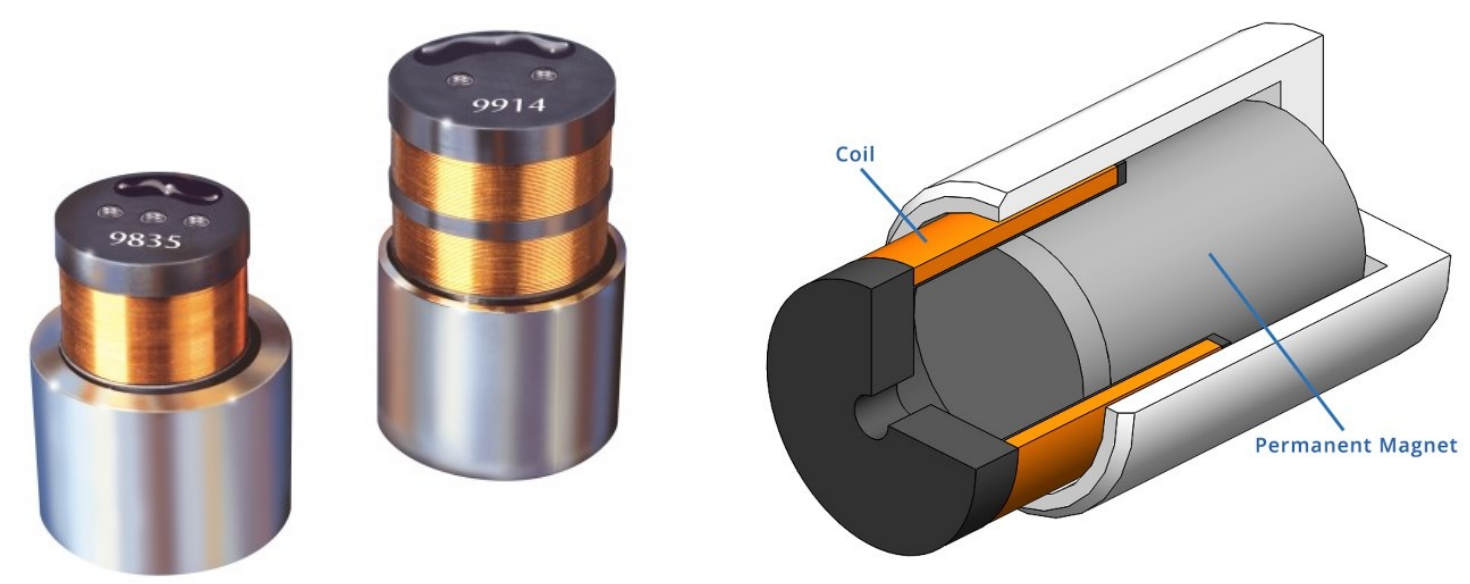

Figure 5: Voice-Coil (left, Bei-Kimco) and Schematic (right, www.powertransmission.com)

\subsection{Vacuum Chamber}

The VEM Drive as well as the torsion balance were placed inside a vacuum chamber with the dimensions of $2.5 \mathrm{~m}$ in length, $1.2 \mathrm{~m}$ in width and $1.5 \mathrm{~m}$ in height. The chamber has 14 ISO-K view ports that are covered with tin foil to dampen the RF signal of the antenna, leaving the chamber. Additionally, numerous flanges of different size are available. Two KF40 flanges are used for the RF and the interferometer feedthrough respectively and one CF63 flange supports two SubD 25 connectors used to connect the balance with its control instruments and computer.

To create a vacuum, a roots pump ECO 3-65+ and a cryo pump CooIVAC iCL by Leybold are connected to the chamber. The pressure is read with a Pfeiffer PKR 361 gauge. The scroll pump can reach a vacuum of $10^{-3} \mathrm{mbar}$ and the cryo pump $10^{-8} \mathrm{mbar}$, given sufficient time. 


\section{Test Setup}

This section describes the different experimental setups performed during the test campaign. Seven different setups were built to determine a possible influence from air, RF-/thermal radiation, distance between antenna and target, angle of attack and antenna configuration.

\subsection{Setup 1 - Main Setup}

Setup 1 (Figure 6) consists of antenna 1, its mounting platform, the torsion balance and the POM target. The antenna is fixed to the platform via zip ties. The distance between the POM target and the root of the antenna amounts to $1130 \mathrm{~mm}$. The antennas root also has a distance of $260 \mathrm{~mm}$ to the side of the vacuum chamber and $882 \mathrm{~mm}$ to the back. The center of the target, as well as the center of the antenna is $430 \mathrm{~mm}$ above the floor of the chamber.

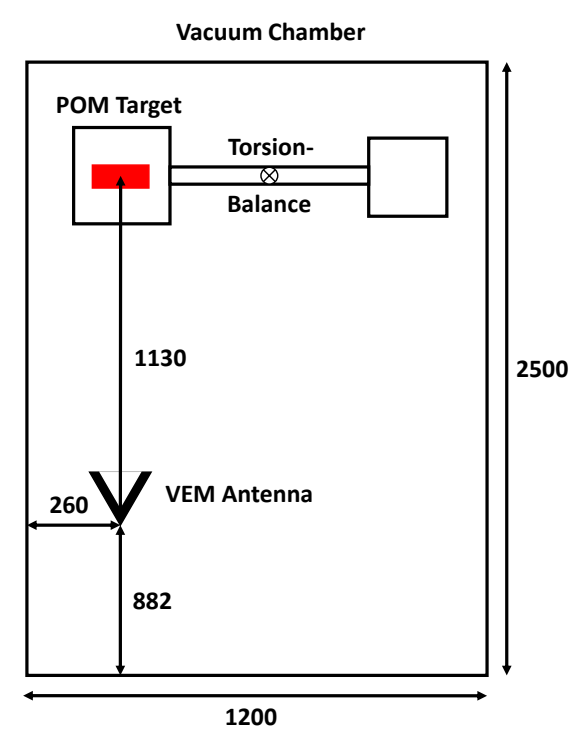

a) Schematics

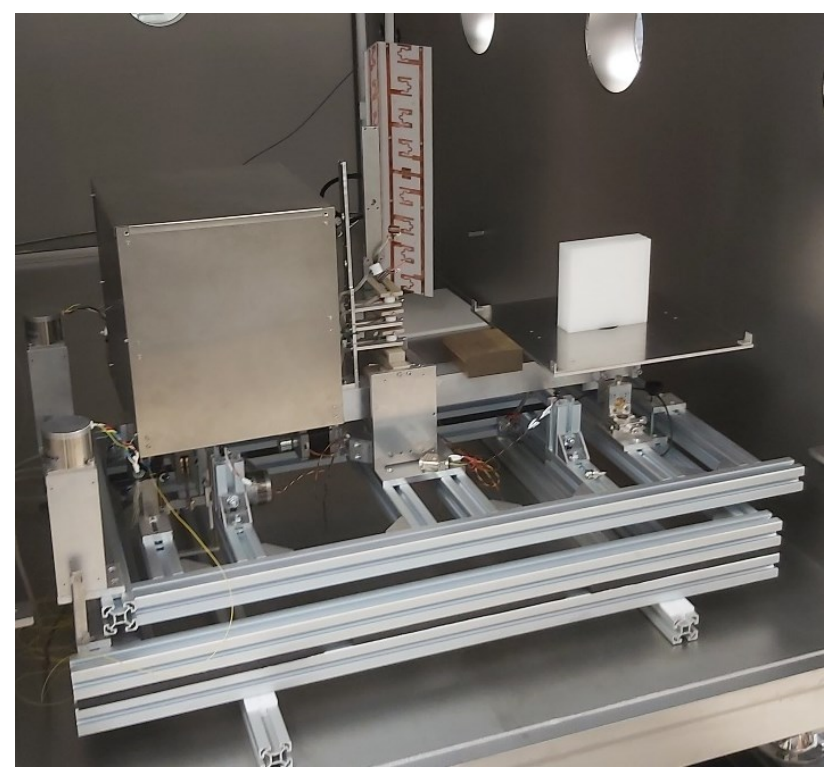

b) Actual setup

Figure 6: Setup 1 - main setup

\subsection{Setup 2 - Main Setup + Vacuum}

Setup 2 was done to determine a possible influence of air. In low pressure environments, air movement caused by convection is mitigated. It is exactly the same as setup 1, except the vacuum chamber is evacuated and has a pressure of $2.1 \times 10^{-6} \mathrm{mbar}$.

\subsection{Setup 3 - Main Setup + Shielding}

Setup $3 a$ and $3 b$ was done to determine, if a possible effect can be destroyed by shielding the antenna from the target on the torsion balance.

\subsubsection{Setup 3a - Metal Shielding}

Setup 3a (Figure 7) introduces a steel grid between the antenna and the torsion balance. The remaining holes between the grid and the vacuum chamber are covered with tin foil. The grid consists of steel wires with a diameter of $0.06 \mathrm{~mm}$ and a mesh size of $0.6 \mathrm{~mm}$. The tin foil is taped to the grid and the walls of the chamber with Kapton tape to ensure an electrical connection. 


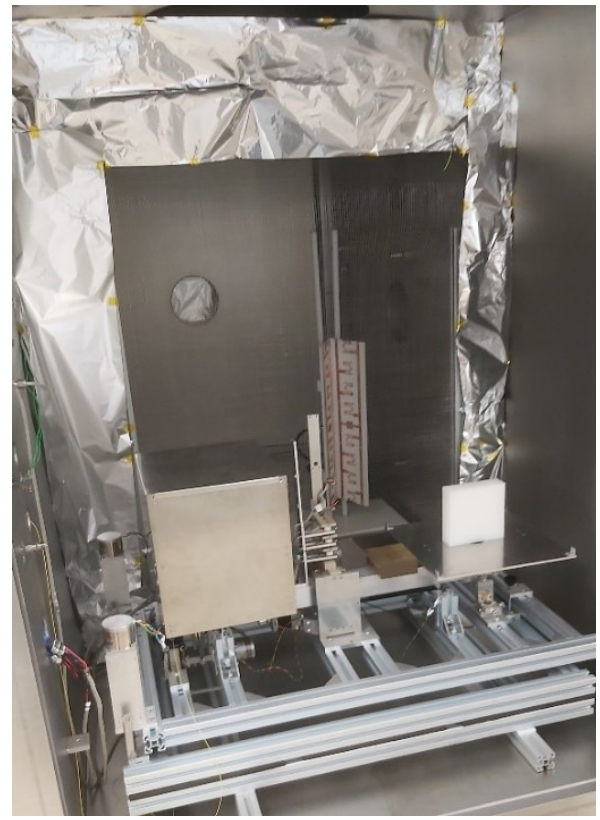

Figure 7: Setup 3a- metal shielding with steel grid + tin foil

\subsubsection{Setup 3b - Thermal Shielding}

Setup $3 b$ (Figure 8) consists of an extruded polystyrene plate (also called XPS, or styrodur) between the antenna and the balance. XPS has a low thermal conductivity and can shield the balance from potential thermal radiation from the antenna. The plate is not large enough to separate the antenna and the balance completely.

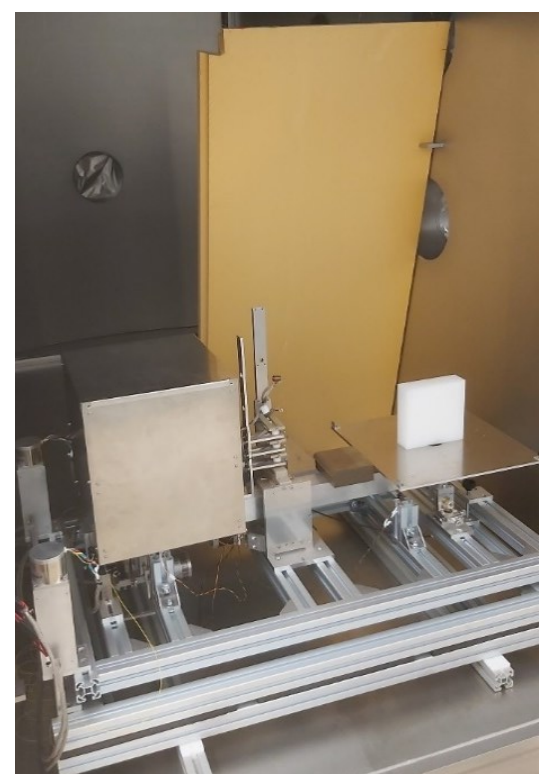

a) Front view

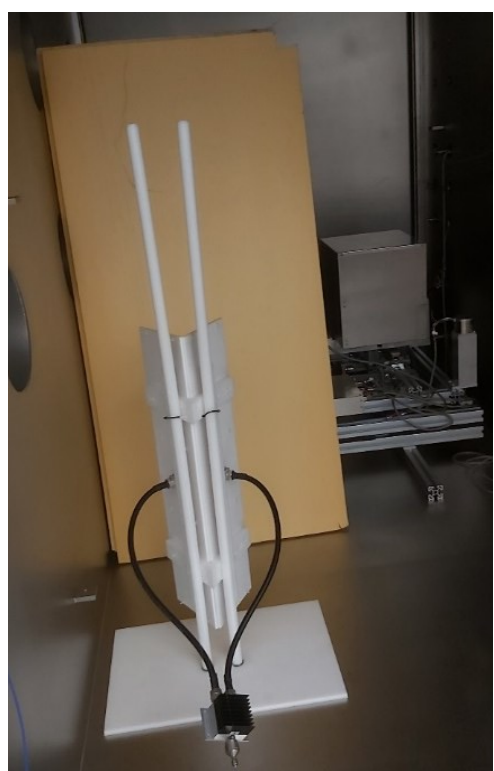

b) Rear view

Figure 8: Setup 3a - thermal shielding with XPS 


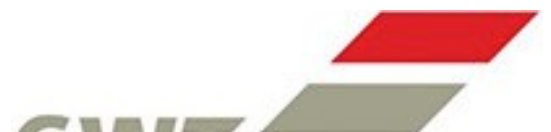

forschung+innovation
Doc: VEM-GWT-TN-1001

Issue: 1

Date: 5th March 2020

\subsection{Setup $4-520 \mathrm{~mm}$}

In setup 4 the distance between the antennas root and the target on the balance is lowered to $520 \mathrm{~mm}$. This is achieved by moving the balance closer to the antenna. The antenna itself is not moved, to avoid changing the RF-configuration of the cables and antenna itself.

\subsection{Setup 5 - Angle $90^{\circ}$}

Setup 5 (Figure 9) has the antenna rotated $90^{\circ}$ so it is facing the side wall of the vacuum chamber. Due to the long and stiff cables, connecting the antenna and the splitter, the antennas root changed its position. It is $329 \mathrm{~mm}$ away from the wall of the chamber and $795 \mathrm{~mm}$ from the back. The distance to the balance changed as well to $607 \mathrm{~mm}$.

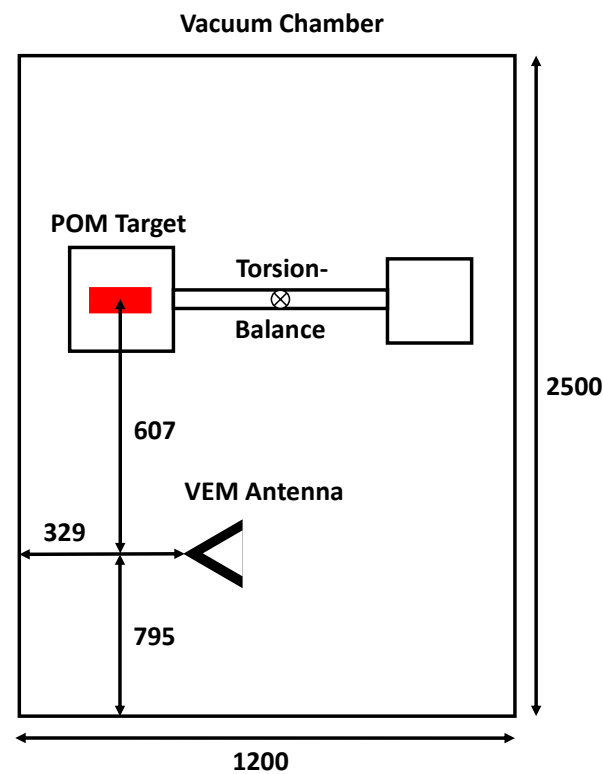

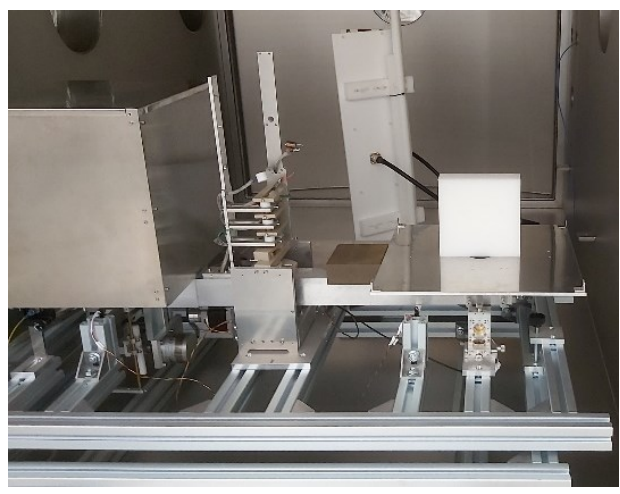

b) Actual setup

a) Schematics

Figure 9: Setup 5 - antenna turned $90^{\circ}$

\subsection{Setup 6 - Angle $15^{\circ}$}

Setup 6 (Figure 10) has the antenna pointing towards the target at an angle of $15^{\circ}$ and also has the steel grid with tin foil placed between the antenna and the balance. The distance to the side of the chamber increased to $555 \mathrm{~mm}$. The distance to the back did not change. 


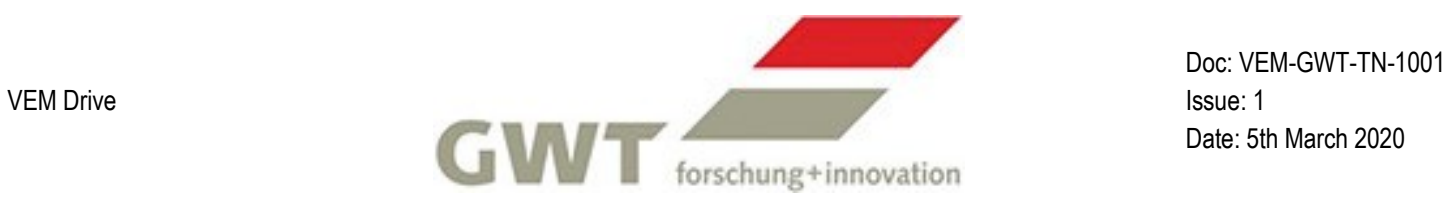

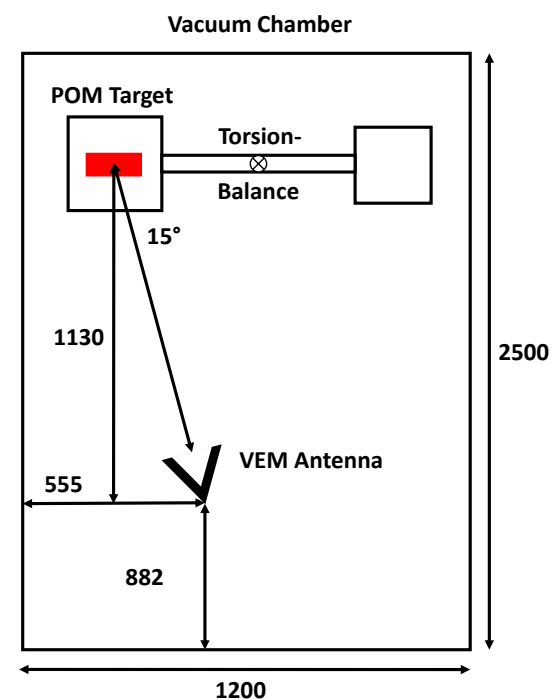

a) Schematics

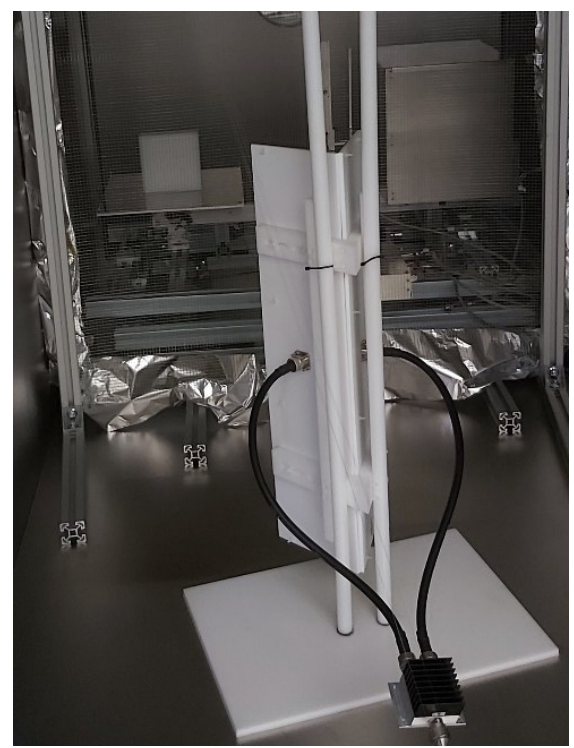

b) Actual setup

Figure 10: Setup 6 - angle of attack $15^{\circ}$

\subsection{Setup 7 - Main Setup + Antenna 2}

Setup 7 has the same parameters as setup 1, except it uses antenna 2 with a different configuration of the fractal metal-geometry. 


\section{RESULTS}

This section will present the results of the test campaign. First, it will describe the calibration of the balance and then the results for each setup.

\subsection{Balance Calibration}

The balance was calibrated after the antenna was in place and all RF tests, described in section 2.1.3, were finished. Figure 11 shows the result of the calibration that gives a conversion factor between displacement and force of $\mathrm{K}=1.86 \mu \mathrm{N} / \mu \mathrm{m}$.

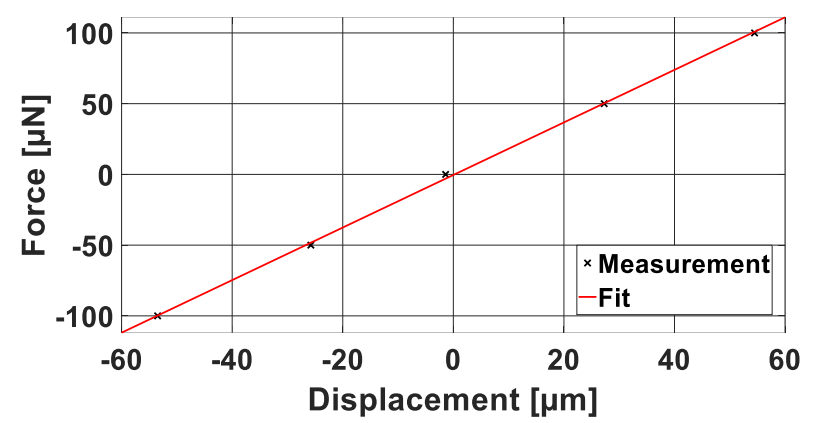

Figure 11: Balance calibration after evacuation of chamber with $\mathrm{K}=1.86 \mu \mathrm{N} / \mu \mathrm{m}$

For setups that did not change the position of the balance, only a very quick check of the calibration factor was done. A force was commanded and compared to the measured force. When both were the same, no complete calibration was performed again. However, after changes to the balance or the environment (evacuating the chamber) a complete calibration was performed. The factor at the beginning of the campaign was $2.10 \mu \mathrm{N} / \mu \mathrm{m}$, which changed during the vacuum test to $1.86 \mu \mathrm{N} / \mu \mathrm{m}$. All measurements presented here were performed after the vacuum test, so the force was calculated using $K=1.86 \mu \mathrm{N} / \mu \mathrm{m}$. 


\subsection{Force Measurements}

Each setup was tested at different power levels. A representative force measurement is shown in Figure 12 for setup 1 (distance antenna-balance of $1130 \mathrm{~mm}$ and on air). A power pulse to the VEM antenna caused a corresponding movement of the balance that is converted into thrust. For the force measurement, the average of the second half of the power pulse is used after the balance movement settled. A table next to the graph lists the force and reflected power at the circulator for each commanded power to the antenna. Because the circulator power is very low, the antenna was working well at its resonance. The measured force of the balance increased with higher power levels. The positive force means that the POM target moves away from the antenna. Although the force was rather small (in the $\mu \mathrm{N}$ range compared to expected fractions of a Newton), the fact that we do measure something was surprising.

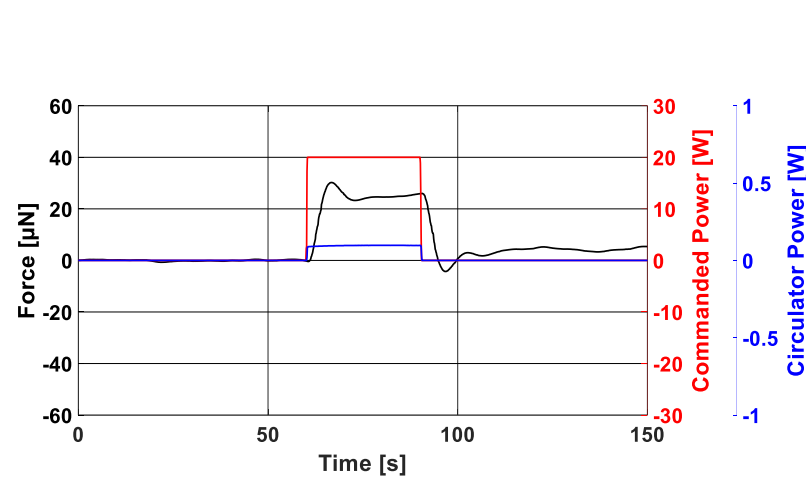

Figure 12: Results setup $1-20$ W

\begin{tabular}{|r|r|r|}
\hline $\begin{array}{l}\text { Com. Power } \\
{[\mathrm{W}]}\end{array}$ & \multicolumn{1}{|c|}{$\begin{array}{c}\text { Force } \\
{[\mu \mathrm{N}]}\end{array}$} & \multicolumn{1}{c|}{$\begin{array}{c}\text { Circ. Power } \\
{[\mathrm{W}]}\end{array}$} \\
\hline 5 & 7.2 & 0.0 \\
\hline 10 & 14.0 & 0.1 \\
\hline 15 & 24.0 & 0.1 \\
\hline 20 & 25.0 & 0.1 \\
\hline 25 & 30.0 & 0.1 \\
\hline 30 & 40.0 & 0.1 \\
\hline 35 & 41.0 & 0.1 \\
\hline 40 & 49.0 & 0.1 \\
\hline 45 & 50.0 & 0.1 \\
\hline
\end{tabular}

Table 2: Results setup 1 (main)

Figure 13 shows a comparison of this setup against different distances and angles. All measurements are quite similar within a certain spread - with the exception of the antenna position at $90^{\circ}$. However, later analysis revealed that this was not due to the position but due to a setting on our computer program, which forced the voice-coil actuator to remain at zero current (see next section).

Next, Figure 14 shows a comparison of the force values for different power level in different environments, including high vacuum, a metal grid or thermal shield between antenna and balance as well as the second antenna.

The surprise here was that the metal grid, which should shield electromagnetic radiation between antenna and balance, actually nearly doubled the measured force. The radiated power of the antenna outside the chamber was measured using the spectrum analyzer and it was $10 \mathrm{~dB}$ lower compared to the measurements without the shield. The thermal shield resulted again in a zero-measurement, again due to a different voice-coil setting on the computer program.

We therefore concluded that the observed force is most likely a facility artefact and not due to an electromagnetic (rules out with metal grid shield) or thermal interaction (ruled out with vacuum). 


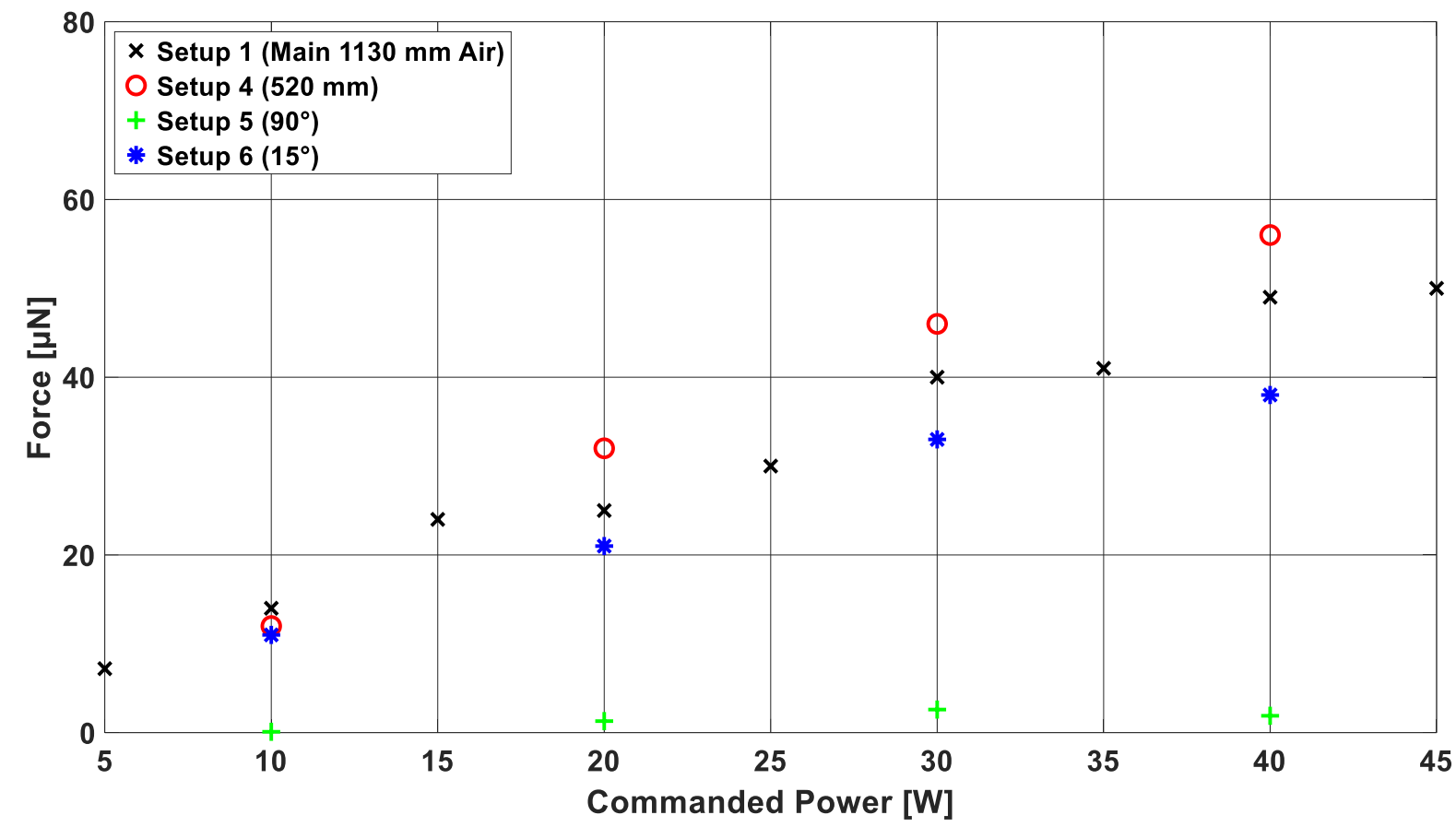

Figure 13: Comparison of Different Distances and Angles - All Measurements in Air

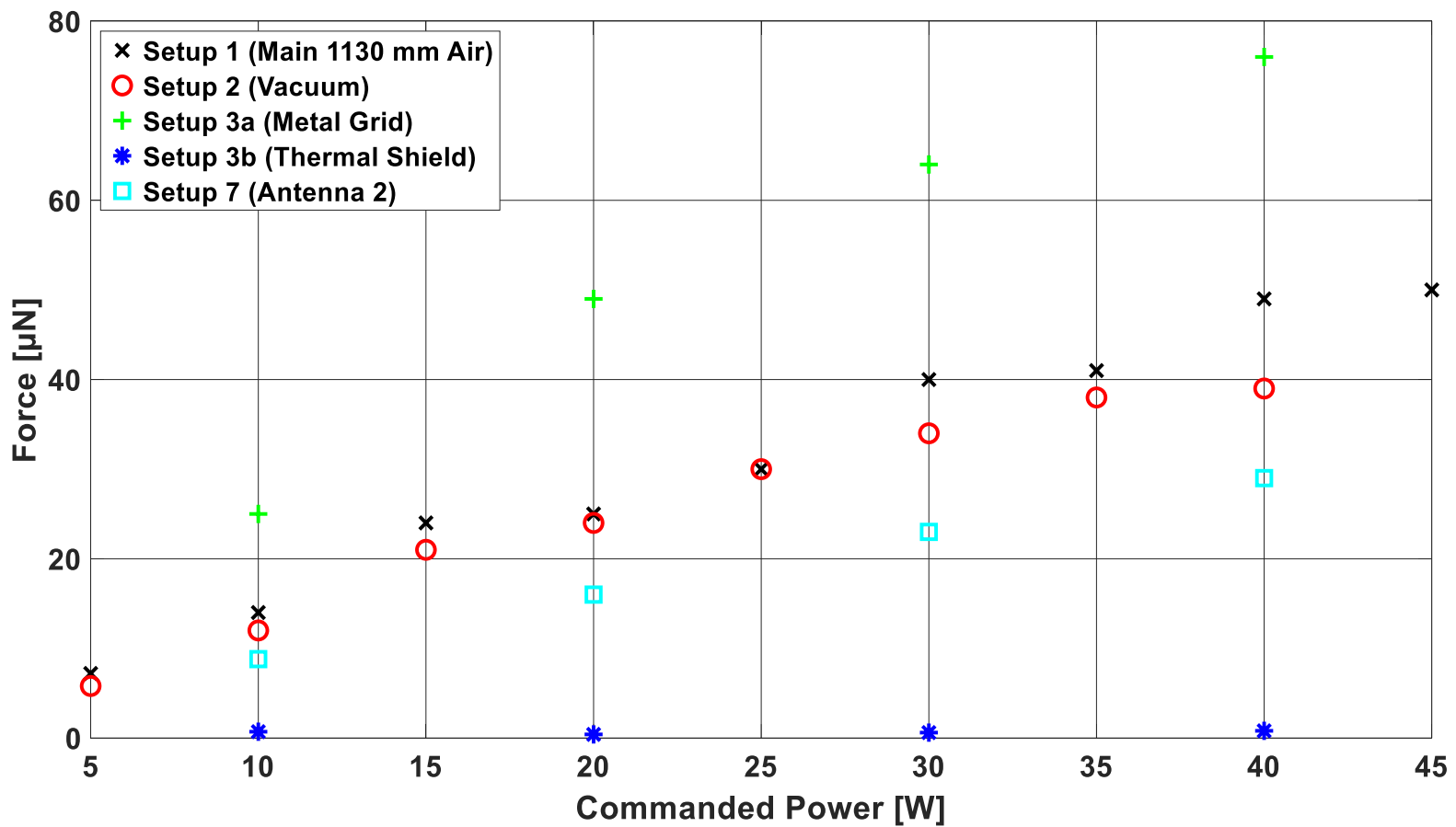

Figure 14: Comparison of Different Measurement Conditions with same Distance 


\subsection{Voice Coil Influence Measurements}

As already mentioned in the section before, the voice coil (the calibration device of the balance) seems to have an influence on the measurements. Usually it does not matter if the power supply is on or off because a command of zero-current is equivalent of a zero-force on our calibrator.

However, the DC power supply has most likely a diode in its internal circuit and therefore can rectify an $\mathrm{AC}$ signal that is picked up by the coil. This can easily be tested by e.g. changing the polarity of the voice coil connections which should then also reverse the observed force.

This lead to the following tests:

- A) Setup $1+$ cable of voice coil normal (positive)

- B) Setup $1+$ cable of voice coil flipped (negative)

- C) Setup $1+$ cable of voice coil disconnected and "open circuit"

- D) Setup $1+$ cable of voice coil disconnected and "shortened circuit"

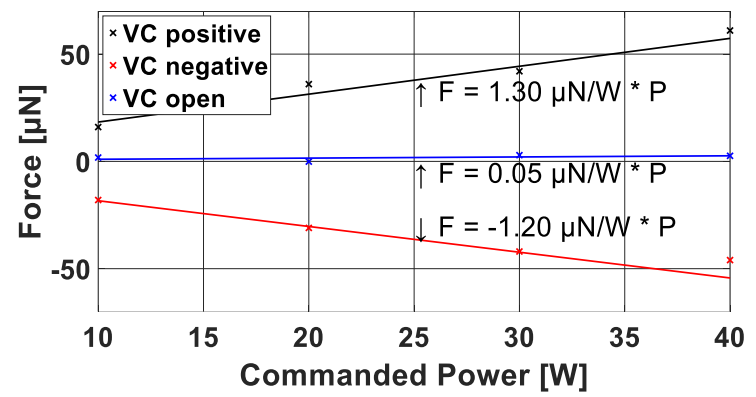

Figure 15: Comparison of voice coil results (fits do not

\begin{tabular}{|l|r|r|}
\hline Test & \multicolumn{1}{|c|}{$\begin{array}{c}\text { Force } \\
{[\mu \mathrm{N}]}\end{array}$} & \multicolumn{1}{c|}{$\begin{array}{c}\text { Circ. Power } \\
{[\mathrm{W}]}\end{array}$} \\
\hline $\mathrm{A}$ & 42.0 & 0.1 \\
\hline $\mathrm{B}$ & -42.0 & 0.1 \\
\hline $\mathrm{C}$ & 2.9 & 0.1 \\
\hline $\mathrm{D}$ & -1.7 & 0.1 \\
\hline
\end{tabular}

Table 3: Voice coil results for $30 \mathrm{~W}$ commanded power include force of $40 \mathrm{~W}$ )

Figure 15 and Table 3 show the results of these tests. It can be clearly seen that the direction of the force indeed changed, by reversing the connection of the voice coil. In addition, if the power supply is disconnected, the force vanishes as well (no diode to rectify a signal in the loop any more). Figure 16 shows a measurement of the induced voltage in the voice coil for $1.0 \mathrm{~W}$ commanded power to the antenna. The voice coil picks up the commanded frequency of $146.0 \mathrm{MHz}$ with a voltage amplitude of $0.3 \mathrm{~V}$.

This voice coil test showed, that all previous measurements (setup 1 to 7) were all artifacts caused by the voice coil.

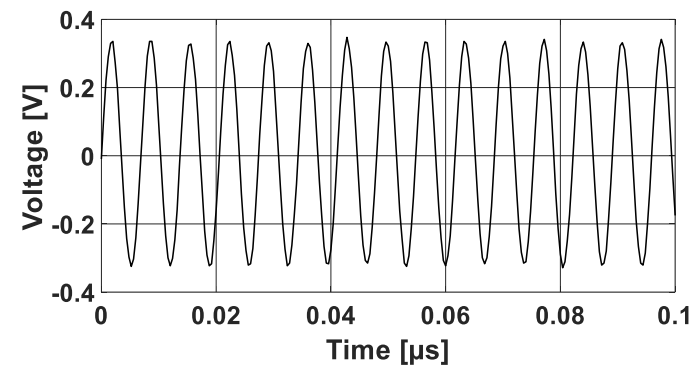

Figure 16: Oscilloscope measurement of voice coil for $1 \mathrm{~W}$ commanded power 


\subsection{Additional Remarks}

After antenna 1 was removed from the chamber, burn marks were spotted on the copper parts of the antenna. These spots are located at the top and bottom parts and were most likely caused during the higher power levels.

Antenna 2 showed no such marks, but was also used less than antenna 1.

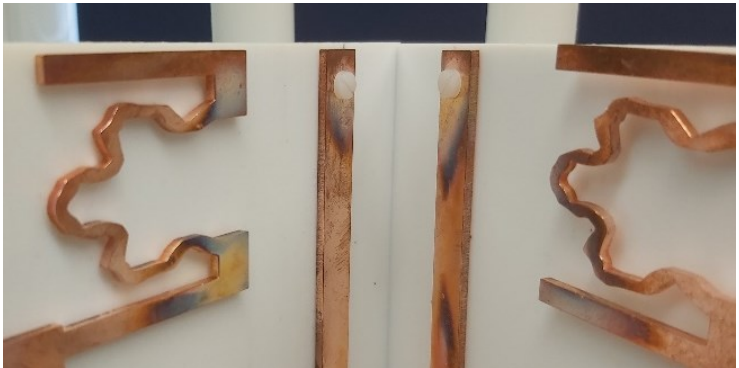

a) Top part

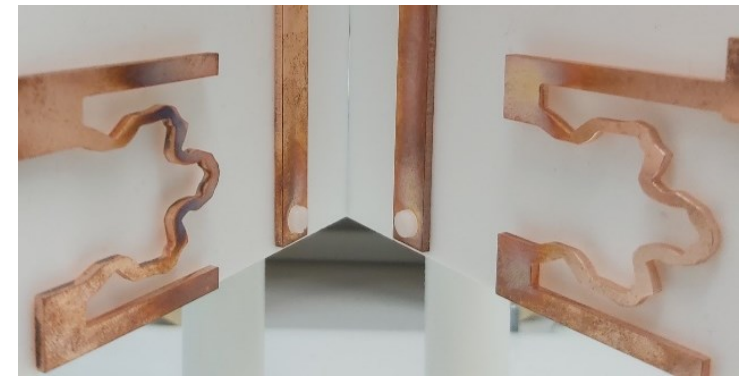

b) Bottom part

Figure 17: Burn marks on antenna 1

Burn marks were also spotted at the bottom of the vacuum chamber, around the base the antenna mount (Figure 18). They are pointing towards the balance.

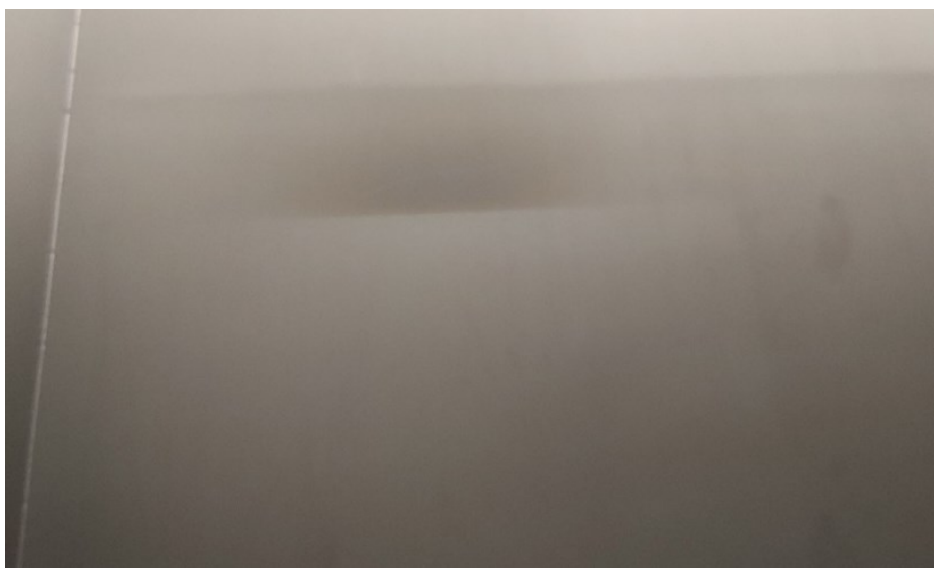

Figure 18: Burn marks around the antenna 


\section{CONCLUSION}

A VEMDrive at a resonance frequency of $146 \mathrm{MHz}$ within the VHF band was tested with power levels up to $45 \mathrm{~W}$ inside a large vacuum chamber that allowed sufficient space for testing inside and blocked the radiation to the outside environment. $\mathrm{A} \mu \mathrm{N}$-resolution thrust balance with a POM target mass was used to determine if a force from a claimed spacetime-distorsion can be measured. The balance used a laser interferometer to determine the force and a voice-coil actuator for calibration purposes.

Initially, forces in the range of $1 \mu \mathrm{N} / \mathrm{W}$ were seen, which is orders of magnitude below claimed values [1]. No particular influence on distance and orientation as well as different environments (vacuum, air or shields) were found. However, some measurements showed zero forces if the calibrator's power supply was turned on although at zero command. This led us to suspect that the coil of the calibrator picks up the high frequency radiation. If the power supply is turned on, internal diodes rectify this AC signal into a DC output which in turn caused the balance to move. By changing the polarity of the coil and by disconnecting it from the power supply, the observed force was able to flip polarity too and eventually vanish.

We therefore conclude that the VEMDrive does not produce any anomalous forces at its resonance frequency of $146 \mathrm{MHz}$ and within our tested power levels and balance resolution. Our measurements set a limit of $0.050 \mu \mathrm{N} / \mathrm{W}$ for any anomalous forces (compared to claimed $3780 \mu \mathrm{N} / \mathrm{W}$ [1]) - some 6 orders of magnitude below. This is just an order of magnitude higher than standard radiation pressure and therefore even if a force exists in that range, it would not be better than current state-of-the-art.

In any case, the influence of a voice-coil calibration in combination with a RF environment is certainly of interest and should be taken into account for similar measurements. A simple relay, which disconnects the calibrator during measurements, is advisable to eliminate this problem. 
VEM Drive

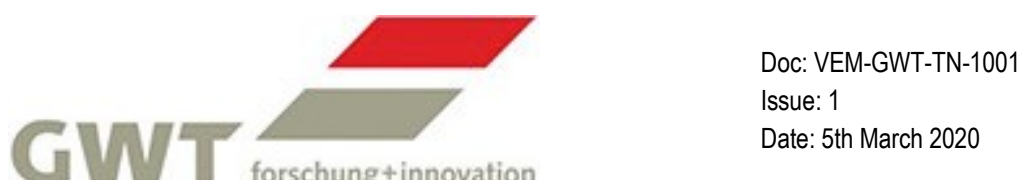

\section{References}

[1] D. P. Pares and K. Finley, "The artificial inducement of a local space warp bubble using a VEM drive," AIAA Joint Propulsion Conference, AIAA 2018-4633, 2018.

[2] M. Kößling, M. Monette, M. Weikert, and M. Tajmar, "The SpaceDrive project - Thrust balance development and new measurements of the Mach-Effect and EMDrive Thrusters," Acta Astronaut., vol. 161, no. May, pp. 139-152, 2019. 